\title{
Organizar el espacio público para el distanciamiento social y la complementariedad modal: metodología y aplicación
}

\section{Organizing Public Space for Social Distancing and Modal Complementarity: A Methodology and Application}

Óscar SÁNCHEZ-FLORES

Facultad de Ingeniera Benemérita Universidad Autónoma de Puebla | México Contacto: oscar.sanchezflores@correo.buap.mx

Yeraldin ROMERo

Departamento de Sistemas de Información Geográfica

GENIE S. C.

Contacto: yeraldin.romero@genie.org.mx

\section{Resumen}

El artículo explora la factibilidad y las ventajas de reorganizar el espacio público disponible para atender las problemáticas de movilidad y que se propicien condiciones para respetar el distanciamiento social. Se desarrolla una metodología para reorganizar el espacio público, entendido aquí como el espacio para la movilidad con vehículos de motor en áreas urbanas consolidadas, el propósito consiste en favorecer el distanciamiento social a partir del enfoque de propiciar el uso modal mixto del espacio disponible. En primer lugar se delimita el área a intervenir y se elabora el inventario de las características geométricas del espacio público disponible. Posteriormente, se identifica la tipología predominante de las secciones transversales con base en sus características geométricas. Enseguida, se proponen las secciones transversales tipo. Estas promueven el uso modal mixto del espacio público tomando en cuenta tanto las dimensiones modales mínimas para la circulación como la jerarquía de la movilidad sostenible. En este dimensionamiento se prioriza el distanciamiento 
social a través de la asignación de espacio homogéneo y suficiente para los desplazamientos peatonales. Finalmente, las secciones, caracterizadas por un uso modal mixto, se van alineando con las vocaciones o usos de suelo predominante del entorno urbano. A este nivel de definición se incorporan los espacios requeridos para la accesibilidad universal tanto para el acceso de los inmuebles como para los cruces seguros. Adicionalmente, se definen indicadores para determinar el efecto de la reasignación del espacio público sobre el distanciamiento social y la movilidad. La metodología se aplica a un centro histórico del centro del México en la confluencia de residentes y externos para realizar sus actividades diarias. Con herramientas geográficas se presenta la variación de los indicadores con y sin intervención para mejorar el distanciamiento social. Los resultados muestran una mejora importante en el distanciamiento social disminuyendo el riesgo de exposición y contagio durante la pandemia del coronavirus.

Palabras clave: pandemia, distanciamiento social, espacio público, movilidad durable, complementariedad modal

\begin{abstract}
This article develops a methodology to reorganize public space in urban areas. Mixed modal use of the available space approach is proposed to stimulate social distancing. First, the study area is delimited, and an inventory of the geometric characteristics in the available public space is drawn up. Secondly, based on their geometric characteristics, the predominant typology of the cross-sections is identified. Next, new standard cross-sections are proposed. These promote the mixed modal use of public space, considering both the minimum modal dimensions to circulate and the hierarchy of sustainable mobility. In this order, social distancing is prioritized by allocating homogeneous and sufficient space for pedestrian movements. Finally, characterized sections by a mixed modal use are aligned with the vocations of predominant land uses of the urban environment. At this level, accessibility criteria are included both for access to buildings and for safe crossings. Furthermore, key indicators to measure the effect for which the reallocation of public space has in social distancing and mobility terms are defined. The proposed methodology is applied in a historic Central Business District of Mexico (CBD). In this CBD, many people come together to carry out their daily activities. Geographic tools are used to show as social distance improves when the study zone has an intervention. The case studied results show a significant improve-
\end{abstract}


ment in social distancing, thereby reducing the risk of exposure and contagion during the coronavirus SARS-COV2 pandemic.

Keywords: pandemic, social distancing, public space, sustainable mobility, modal complementarity

\section{Introducción}

La pandemia ocasionada por el coronavirus SARS-COV-2 ha generado cuantiosos daños irrecuperables tanto en vidas humanas como en una amplia cantidad de sectores económicos y sociales a escala global y local. Los efectos que se derivan de las distintas acciones comunitarias para evitar la propagación del virus se han presentado de forma inmediata en algunos sectores, como salud. En otros casos, están catalizando transformaciones en la sociedad propias de los nuevos modelos de producción asociados con la tecnología, la energía y las telecomunicaciones. La irrupción del virus ha propiciado cambios que a su vez repercutirán en otras actividades, marcando un hito en la sociedad y posiblemente una nueva era denominada Poscovid. Una de las acciones de la estrategia para contener la propagación del virus, adoptada por la mayoría de los países, ha sido la restricción de la movilidad y el distanciamiento social. Sin embargo, la implementación de esta última medida encuentra dificultades operativas cuando se trata de espacios públicos en el que las condiciones físicas y geométricas corresponden a un modelo de producción y circulación en el que necesariamente confluyen una gran cantidad de personas. Por otro lado, las restricciones a la movilidad difícilmente pueden mantenerse por periodos prolongados ya que afectan al modelo productivo existente. Dada las limitaciones de capacidad de cualquier espacio público, se plantea el uso modal mixto en el que los modos de transporte se complementan siguiendo el paradigma de la jerarquía de la movilidad sostenible. Para tal efecto, primero, se realiza una revisión de los conceptos de espacio público y distanciamiento social, que son el marco teórico sobre el cual se desarrolla la metodología que se plantea. Posteriormente, se describen los componentes de ésta y se desarrollan indicadores para cuantificar los efectos de la reorganización del espacio público. Finalmente, se ilustra una aplicación empírica con el propósito central de elaborar una metodología para la reordenación total del espacio público en zonas saturadas de traza ortogonal, para las 
distintas formas de movilidad de manera que pueda emplearse en estas condiciones extremas de pandemia, donde persiste el confinamiento para evitar contagios.

\section{¿Qué es el espacio público?}

En principio el termino espacio se trata en este artículo desde la perspectiva de la geografía crítica humanista (Balaguer, 2018) en la que "el espacio se define como un conjunto de formas representativas de relaciones sociales del pasado y del presente y por una estructura que queda expresa en las relaciones sociales, a su vez indicativas de procesos y funciones" (Milton Santos en Hiernaux y Lindón, 1993: 103). Para "caracterizar un espacio geográfico en estos términos, implica entenderlo como una forma, como un resultado objetivo de la interacción de múltiples fuerzas en el curso de la historia. La configuración geográfica o configuración espacial es la manera o arreglo espacial que adoptan los objetos que integran el espacio" (Hiernaux y Lindón, 1993: 104). Esta configuración geográfica o configuración espacial es la esencia de dichos objetos en la medida que responden a los procesos sociales que a su vez se expresan como acciones que se concretan a través de formas espaciales. Los procesos sociales adquieren un carácter geográfico porque las acciones sociales se territorializan. En esta territorialización, el movimiento social se fija en un espacio y un tiempo. De esta manera, "la formación de un espacio supone la acumulación de acciones localizadas en diferentes momentos" (Milton Santos en Hiernaux y Lindón, 1993: 104).

La sociedad como totalidad está integrada por diversas instancias o dimensiones, el espacio es una de estas instancias que, por su naturaleza material, capaz de perdurar más que la sociedad que produce las formas espaciales, tiene la posibilidad de influir sobre esa totalidad social. Con ello, el espacio pasa a ser condicionante de los procesos sociales.

La geografía crítica destaca por dos consideraciones esenciales. Una es el antecedente o la historia en la que el espacio, a través de sus formas, sobrevive a los modos y modelos de producción. La otra es la condicionante que el espacio significa para el funcionamiento social global. Se podría afirmar que el pasado deviene en presente a través de las formas espaciales (Hiernaux y Lindón, 1993: 104). 
El espacio se constituye en testigo de un momento en un modo de producción, a través del espacio construido de las cosas fijadas, se crean formas que se deshacen a medida que se desarrollan nuevos procesos, o nuevas instancias, "las estructuras sociales, la particularización de las relaciones de agentes y entre éstos y los elementos naturales (mediados socialmente), está condicionada por las configuraciones espaciales preexistentes" (Coraggio, 1987: 298). Dos características adicionales a este concepto de espacio son, su valor y la inercia dinámica que va a generar las diferenciaciones en su proceso de cambio haciéndolos únicos. Con el beneficio del suelo edificado, "a partir de la renta del suelo, puede analizarse la valorización del espacio" (Moraes, 1984: 126), aunado a la interacción de múltiples fuerzas presentes que condicionan o determinan los procesos sociales futuros creando inercias dinámicas que se manifiestan de distintas maneras. El espacio es, en este sentido, resultado y condicionante, una contradicción entre lo efímero y lo estable, señalan Hiernaux y Lindón (1993). El espacio puede ser subdivido y fragmentado para el ejercicio del poder, lo que impide diversas formas de libre albedrío, facilitando la imposición de políticas en las decisiones sociales, económicas $\mathrm{y}$ territoriales.

Respecto al espacio público del que nadie puede ser excluido o limitado en su acceso y uso, porque es históricamente inherente a la vida social,

Max Weber indicó que la condición primitiva del espacio público [en Europa] se remonta a las plazas de la ciudad medieval europea, caracterizadas por su actividad comercial. Es aquí donde tuvo lugar la forma primitiva de vida pública en el sentido sociológico siendo una situación en la que predomina el anonimato, donde los individuos tienen la libertad de establecer contacto o distanciarse según su voluntad. (Schlack, 2007: 25)

Actualmente se requieren regulaciones, o conjuntos de reglas y normas para evitar o limitar los excesos y desviaciones de algunos actores que pudieran generar afectaciones a terceros. De esta manera, son características del espacio público la integración parcial, el sistema social abierto, la interacción entre desconocidos, así como su grado de accesibilidad y la posibilidad de su uso sin restricciones para cualquier persona. 


\section{El espacio público y el distanciamiento social}

El espacio público define la calidad de la ciudad, porque indica la calidad de vida de la gente y la calidad de la ciudadanía y de sus habitantes (Borja y Muxí, 2003). Cuanto más abierto esté a todos, el espacio público expresará la democratización política y social. La irrupción del coronavirus SARS CoV-2 ha venido a trastocar este concepto de apertura, acceso y calidad de vida del espacio público. En efecto, una de las medidas de mitigación comunitaria de la propagación de la epidemia recomendadas por la Organización Mundial de la Salud (OMS) ha sido el distanciamiento social. Aunque es un constructo social, esta intervención impuesta como política pública derivada de la emergencia epidemiológica debe considerarse como una norma a adoptar desde nuestra conciencia colectiva ante la ausencia de intervenciones de prevención primaria, esto es vacunas, o terapéutica, fármacos antivirales (Lazcano y Alpuche, 2020). Es recomendable que esta implementación de acciones comunitarias se realice sin coerción, con transparencia, con compromiso social y veracidad, pero también con oportunidad y liderazgo. La claridad y certidumbre son elementos críticos para preservar la confianza y disminuir la preocupación social en la reorganización del espacio público, principalmente en las zonas de mayor concentración de personas. Estas corresponden generalmente a zonas donde confluyen diversas actividades económicas, tales como el abastecimiento de mercancías, comercios, restaurantes; la prestación de servicios bancarios, hospitales, transporte, actividades de abastecimiento, con mercados y supermercados, y donde se dispone de una oferta de espacios de entretenimiento, ocio y turismo. Sin embargo, el problema general que se presenta es reorganizar el espacio público para ofrecer condiciones más adecuadas para propiciar el distanciamiento social. Este problema se puede plantear a partir de la gestión de un recurso escaso, con diversos requerimientos de uso, previamente asignado, limitado en su capacidad y sujeto a un conjunto adicional de restricciones de diversa naturaleza.

En los espacios privados el distanciamiento social se ha atendido limitando el número de personas que pueden permanecer en el interior. No obstante, esta acción lleva a la formación de filas y aglomeraciones en banquetas y calles repercutiendo sobre la disponibilidad del espacio público para la movilidad peatonal y, a su vez, afecta la circulación motorizada, las maniobras de carga y descarga de mercancías y las operaciones de ascenso y descenso del transporte público. Es decir, la restricción 
de la movilidad en general presenta un nuevo desafío de asignación del espacio público a fin de garantizar la accesibilidad de bienes y servicios, procurando las mejores condiciones de circulación para todos los modos de transporte. Se utiliza el paradigma de la jerarquización de la movilidad y el desarrollo urbano sostenible, con la asignación de los modos de transporte priorizando a los que promueven la equidad, el beneficio social y producen menos daño ambiental al entorno. Así, la llamada pirámide invertida de la movilidad (ITDP, 2012) establece que las intervenciones para una movilidad sostenible deben priorizar a los peatones, sean infantes, adultos mayores y personas con movilidad reducida, seguido de usuarios de bicicletas y otros modos no motorizados, seguidamente de los pasajeros de transporte público, el transporte de carga y, finalmente, los vehículos particulares (ver Moovit Insights, s. f.). A continuación se esboza la metodología para reorganizar el espacio público procurando el distanciamiento social.

\section{Metodología para la asignación del espacio público para procurar el distanciamiento social}

\section{Delimitar el área a intervenir}

Para facilitar la planeación, control y seguimiento o en su caso "el ejercicio del poder" en democracia, se requiere que el espacio público se subdivida o fraccione. La delimitación de la zona se relaciona con criterios funcionales donde existen aglomeraciones recurrentes y se requiere prever una cierta extensión que permita intervenir para mejorar la circulación de todos los modos de transporte, considerando: 1. La alta afluencia de personas y de movilidad peatonal en los entornos de corredores, zonas comerciales y puntos singulares tradicionales de abastecimiento: mercados y tiendas al menudeo. 2. El desbordamiento de las filas de los establecimientos que ofrecen servicios altamente demandados y que limitan el acceso a las personas al interior de estos recintos. Tal es el caso de bancos, servicios públicos, hospitales, farmacias entre otras. 3. Los entornos en los que existe una alta concentración de usuarios de transporte público ocasionado por el traslape de recorridos de rutas en una misma vía, la densidad de zonas de ascenso y descenso de pasajeros en zonas en particular mercados, calles comerciales, centros de trabajo, entre otros. 
4. La estructura vial y los sentidos de circulación deben permitir tanto la movilidad interna de los modos de transporte como el cruce a través de la zona intervenida sin generar conflictos en el esquema de circulación. Los requerimientos de mayor espacio público para preservar la sana distancia se dan cuando la movilidad confluye en una zona determinada. Tal es el caso de los centros históricos y mercados públicos. Conjuntando espacialmente las zonas, ejes y la estructura vial identificadas se delimita el polígono inicial de intervención en el entendido que debe considerarse cierta flexibilidad para modificarlo en la medida que las restricciones a la movilidad y la variación de la intensidad de los contagios a lo largo del tiempo.

\section{Inventario de las características geométricas del espacio público disponible}

A partir de la cartografía existente de la traza urbana, la información de desarrollo urbano y levantamiento en campo, se obtienen las dimensiones de los anchos de calle, ancho de banqueta, número de carriles, sentidos de circulación, localización de entradas a los inmuebles, zonas de estacionamiento en vía pública, rampas de acceso para la accesibilidad universal, bahías para carga y descarga de mercancías, bahías para ascenso y descenso de usuarios del transporte público entre otras. Esta información se integra en un Sistema de Información Geográfica con sus correspondientes capas.

\section{Tipología de secciones transversales existentes}

La configuración del espacio público es el resultado de una serie de intervenciones previas a lo largo de la historia gestándose usos y vialidades con geometría y dimensiones heterogéneas. Esta condición con frecuencia lleva a la formación de cuellos de botella para la operación y circulación de los distintos modos de transporte. A fin de evaluar la factibilidad de generar espacios más uniformes, es necesario analizar la información de las características geométricas del espacio público disponible para identificar patrones o secciones homogéneas que mantengan la misma geometría y capacidad operativa tan extensa como sea factible. Lo anterior 
se logra constituyendo una tipología de secciones transversales predominantes en la zona intervenida en la que se incluye al menos: cantidad de carriles, ancho de carriles, anchos de banqueta. El análisis de la tipología de las secciones transversales existente permite, en primera instancia, elaborar un diagnóstico tanto de las condiciones actuales de la circulación y movilidad de las personas como de las restricciones físicas que limitan disponer de espacios mínimos de circulación, impidiendo con ello el distanciamiento social recomendable. En segundo lugar, localizar y dimensionar las restricciones físicas, geométricas y de operación existentes a fin de prever las medidas para liberarlas. Siendo estas, por ejemplo: anchos de banqueta reducidas que no cumplan el mínimo requerido, número de carriles disponibles, calles heterogéneas en cuanto a los anchos de banqueta y carriles, con disponibilidad de espacio para bahías de carga, entre otros.

\section{Definición de secciones tipo para un uso modal mixto del espacio público}

Se emplea el término "sección tipo" para identificar la sección transversal de una calle o vialidad que incluye las dimensiones mínimas de las banquetas y sus carriles de circulación. Las dimensiones mínimas corresponden a las normas de diseño de calles vigentes en la zona de estudio o las recomendables en los manuales de diseño. En el caso del distanciamiento social, las dimensiones mínimas se han propuesto conforme a las medidas comunitarias de distanciamiento social indicadas por la Organización Mundial de la Salud. El insumo de entrada para el diseño de estas secciones es la tipología de las secciones transversales existentes las cuales se proceden a analizar para reasignar el espacio público siguiendo los criterios que se indican a continuación. Estas dimensiones mínimas son la base con la que los conceptos de la reorganización del espacio público y las condiciones para la sana distancia que se proponen se materializan. Su incorporación llevará a establecer un conjunto de secciones tipo que reemplazarían a las previas para propiciar el distanciamiento social, a través del cumplimiento de las siguientes cuatro funciones:

1. Ofrecer un espacio homogéneo y suficiente para los usuarios de cada modo de transporte, estableciendo dimensiones mínimas para la circulación, con 
un enfoque de uso mixto del espacio público, en el entendido que difícilmente todos los modos de transporte podrán circular, en condiciones adecuadas, por la misma sección transversal pero también que es espacio público no puede ser monopolizado por un solo modo de transporte. Los conceptos estándar del diseño de calles completas pueden considerarse en la medida de lo posible (véase SEDATU, 2019; NACTO, 2013). Otro aspecto importante es el uso de carriles efectivos para la circulación de cada modo de transporte dimensionando el espacio requerido a favor de los modos no motorizados cuando no aporten alguna utilidad para la circulación.

2. Favorecer el uso mixto del espacio siguiendo la jerarquía de la movilidad sustentable y a la vez propiciando la integración modal. Es decir, esta jerarquía establece las prioridades y el orden de asignación sin demeritar la importancia de la intermodalidad, la complementariedad y la integración horizontal de los modos de transporte.

3. Distribuir espacialmente las operaciones de ascenso y descenso de transporte público. Con esta estrategia se disminuyen las aglomeraciones de este tipo de usuarios, por lo que es recomendable: a) modificar el trazo de las rutas para limitar los recorridos innecesarios al interior de la zona intervenida facilitando la entrada y salida de unidades a través de circuitos, b) limitar la cantidad de unidades que acceden al perímetro de actuación, liberando con ello las vialidades de esta carga vehicular y favoreciendo la circulación al aminorar las maniobras de ascensos y descensos que ocasionan cuellos de botella dinámicos, c) reubicar las paradas de autobuses en espacios públicos amplios y al aire libre separando espacialmente las maniobras de ascenso y de descenso de pasajeros en el caso de las rutas que cierren circuito en la zona intervenida.

4. Asegurar la complementariedad entre las actividades predominantes en entornos espaciales específicos y los modos de transporte. Los usos de suelo predominantes y la presencia de clústers de actividades especializadas, corredores comerciales o turísticos (zonas hoteleras) y proveer espacios para carga, suministro, descarga de mercancías o para maniobras de abastecimiento y operaciones. Para los entornos especializados, es necesario dotar de bahías o zonas de maniobras. 


\section{Configuración del espacio público de uso modal mixto}

Para que las secciones tipo sean operativas se requiere que sean homogéneas y extenderse lo más posible dentro del perímetro de intervención. Para ello, se requiere verificar que la sección tipo elegida es pertinente para toda la calle. Adicionalmente se verificará que corresponda a la vocación o uso de suelo predominante de la misma; por ejemplo, en corredores o ejes comerciales se requiere asignar secciones con bahías para la carga y descarga de mercancías, así como espacio para la circulación peatonal y al menos un carril efectivo para la circulación vehicular de transporte privado. Concluida la asignación las secciones tipo, resta incorporar las entradas, salidas, acceso para personas con movilidad restringida en cada tramo de calle, así como los conceptos de cruces seguros (Fitzpatrick et al., 2005; Stamatiadis y Kirkm, 2010) y la accesibilidad universal.

\section{Proceso de socialización de la intervención e integración} de medidas adicionales a la estrategia de acciones comunitarias

Finalmente, la asignación de secciones tipo para los espacios públicos a intervenir se valida con los diversos actores que confluyen en el área intervenida a fin de incorporar consideraciones omitidas durante el proceso de diseño y se procede a realizar los ajustes necesarios para propiciar la transparencia, certeza y aceptación social referidas como recomendaciones para implementar acciones comunitarias de reducción del riesgo de contagio. Con el objetivo de optimizar el uso del espacio público existente y al mismo tiempo aminorar su subutilización, utilizando la capacidad disponible para disminuir las condiciones de circulación, es conveniente incorporar otras medidas complementarias, las cuales dependerán de las características particulares de cada zona de intervención, incluyendo estacionamiento, en particular para estancias de corto plazo (15-30 minutos), con la finalidad de reducir la circulación redundante en la zona intervenida. Es deseable que todas estas medidas e intervenciones estén contenidas y articuladas en una estrategia pública de acciones comunitarias para reducir el riesgo de contagio, reduciendo la movilidad, procurando no afectar, en la medida de lo posible, las actividades económicas. 


\section{Indicadores de organización del espacio y del distanciamiento social}

Se consideran indicadores pertinentes la oferta de espacios públicos y la relación entre esta oferta y demanda o uso de estos. Los indicadores pueden expresar un valor relativo, cuando se relacionan con la totalidad de oferta en toda la zona de estudio, o absoluto, cuando reflejan un valor en específico. En la relación oferta y demanda pueden ser estáticos o dinámicos según se trate de filas de espera o de circulación de personas. En la Tabla 1 se presenta una síntesis de los indicadores asociados al distanciamiento social en el que se indica además del nombre y tipo la descripción y la fórmula o especificación para obtenerlo.

\section{Tabla 1}

Resumen de indicadores asociados al distanciamiento social

\begin{tabular}{|c|c|c|}
\hline Indicador & Tipo & Descripción \\
\hline $\begin{array}{l}\text { Proporción de Ejes } \\
\text { Modales Mixtos } \\
\text { (PEMM) }\end{array}$ & $\begin{array}{l}\text { Oferta, } \\
\text { relativo }\end{array}$ & $\begin{array}{l}\text { Indica el nivel de } \\
\text { intervención del espacio } \\
\text { público actual que ha sido } \\
\text { modificado para un uso } \\
\text { modal mixto. }\end{array}$ \\
\hline $\begin{array}{l}\text { Proporción de } \\
\text { espacios públicos } \\
\text { ampliados para } \\
\text { uso peatonal } \\
\text { (PEPP) }\end{array}$ & $\begin{array}{l}\text { Oferta, } \\
\text { relativo }\end{array}$ & $\begin{array}{l}\text { Indica el nivel de } \\
\text { disponibilidad de } \\
\text { banquetas con ancho } \\
\text { suficiente para la } \\
\text { circulación y la formación } \\
\text { de filas }\end{array}$ \\
\hline $\begin{array}{l}\text { Superficie } \\
\text { dedicada a la } \\
\text { circulación } \\
\text { peatonal (SCP) }\end{array}$ & $\begin{array}{l}\text { Oferta, } \\
\text { absoluto }\end{array}$ & $\begin{array}{l}\text { Indica la superficie } \\
\text { disponible para uso } \\
\text { peatonal contabilizada a } \\
\text { partir de la superficie de } \\
\text { banqueta disponible }\end{array}$ \\
\hline
\end{tabular}

Fórmula

$P E M M=100 \frac{\sum_{1, M} L_{E M M}}{\sum_{1, N} L_{Z E}}$,

$M:$ total de tramos de ejes modales mixtos,

$N$ : total de tramos de la zona de estudio,

L: Longitud del tramo;

$j=$ EMM: Ejes Modales Mixtos; ZE:

Zona de Estudio

$P E P P=100 \frac{\sum_{1, P} L_{B A P}}{\sum_{1, N} L_{Z E}}$,

$P$ : total de tramos con banquetas ampliadas para circulación y filas $N$ : total de tramos de la zona de estudio, $L$ : Longitud $i ; i=$ BAP: Banquetas Ampliadas para Peatones; ZE: Zona de Estudio

$S C P=\sum_{i=1, N} L_{i} A_{i}$,

$N$ : total de tramos de la zona de estudio,

$L_{i}=$ Longitud de banqueta

del tramo $i$,

$A_{i}=$ Ancho de banqueta del tramo $i$, 
Tabla 1. Continuación

\begin{tabular}{|c|c|c|c|}
\hline Indicador & Tipo & Descripción & Fórmula \\
\hline $\begin{array}{l}\text { Cantidad de } \\
\text { Espacios Vitales } \\
\text { (CEV) para } \\
\text { formación de filas } \\
\text { de espera con } \\
\text { distanciamiento } \\
\text { social }\end{array}$ & $\begin{array}{l}\text { Oferta, } \\
\text { absoluto }\end{array}$ & $\begin{array}{l}\text { Indica la cantidad de } \\
\text { personas que se pueden } \\
\text { formar en filas de espera, } \\
\text { en el espacio público, } \\
\text { considerando un espacio } \\
\text { vital de } d s \text { metros por } \\
\text { distanciamiento social } \\
\text { y } s f \text { metros de separación } \\
\text { entre filas contiguas, }\end{array}$ & $\begin{array}{l}C E V=\sum_{i=1, N} E_{i} K_{i}, \\
N \text { : total de tramos de la zona de } \\
\text { estudio, } \\
E_{i}=\frac{L_{i}}{d s} ; E_{i} \text { es una variable entera o } \\
\text { discreta. } \\
K_{i}=\frac{A_{i}}{s f} ; K_{i} \text { es una variable entera o } \\
\text { discreta. } \\
L_{i}=\text { Longitud de banqueta tramo } i, \\
d s: \text { distanciamiento social } \\
\text { establecido, } \\
s f: \text { separación entre filas contiguas } \\
A_{i}=\text { Ancho de banqueta } \\
\text { tramo i, }\end{array}$ \\
\hline $\begin{array}{l}\text { Densidad de } \\
\text { personas en las } \\
\text { Superficies } \\
\text { dedicadas a la } \\
\text { Circulación } \\
\text { Peatonal (DSCP) }\end{array}$ & $\begin{array}{l}\text { Oferta-de- } \\
\text { manda, } \\
\text { estático }\end{array}$ & $\begin{array}{l}\text { Indica cuantas personas } \\
\text { por unidad de superficie } \\
\text { están ubicadas en las } \\
\text { áreas de circulación } \\
\text { peatonal o banquetas en } \\
\text { periodo de tiempo } \\
\text { determinado. Este } \\
\text { indicador dependerá del } \\
\text { periodo de aforo peatonal. }\end{array}$ & $\begin{array}{l}D S C P_{i}=V_{i} / S C P_{i}, \\
V_{i}: \text { volumen o aforo de personas en } \\
\text { un periodo de tiempo dado en el } \\
\text { tramo } i \text {, } \\
S C P_{i} \text { Superficie dedicada } \\
\text { a la circulación peatonal } \\
\text { del tramo } i,\end{array}$ \\
\hline $\begin{array}{l}\text { Concentración de } \\
\text { personas en las } \\
\text { superficies } \\
\text { dedicadas a la } \\
\text { Circulación } \\
\text { Peatonal (CSCP) }\end{array}$ & $\begin{array}{l}\text { Oferta- } \\
\text { demanda, } \\
\text { dinámico }\end{array}$ & $\begin{array}{l}\text { Indica cuantas personas } \\
\text { por unidad de distancia se } \\
\text { encuentran en las áreas de } \\
\text { circulación peatonal o } \\
\text { banquetas en un instante } \\
\text { de tiempo dado. }\end{array}$ & $\begin{array}{l}C S C P_{i}=\frac{q_{i}}{K_{i} u_{i}}, \\
q_{i}: \text { flujo peatonal en el tramo } i, \\
K i=\frac{A_{i}}{s f} ; K_{i} \text { es una variable entera o } \\
\text { discreta. } \\
A_{i}=\text { Ancho de banqueta del tramo i, } \\
s f: \text { separación entre filas contiguas, } \\
u_{i}=\text { velocidad de caminata } \\
\text { promedio, del tramo i, }\end{array}$ \\
\hline $\begin{array}{l}\text { Distanciamiento } \\
\text { Social Promedio } \\
\text { (DSP) }\end{array}$ & $\begin{array}{l}\text { Oferta- } \\
\text { demanda, } \\
\text { dinámico }\end{array}$ & $\begin{array}{l}\text { Es la separación promedio } \\
\text { entre las personas } \\
\text { considerando una } \\
\text { distribución homogénea } \\
\text { en la circulación }\end{array}$ & $\begin{array}{l}\frac{1}{C S C P_{i}}, \\
C S C P_{i}: \text { Concentración de personas } \\
\text { en las superficies dedicadas a la } \\
\text { Circulación Peatonal del tramo } i \text {, }\end{array}$ \\
\hline
\end{tabular}

Fuente: Elaborado por los autores. 
Los indicadores pueden ser obtenidos para un tramo o conjunto de tramos que conforman el espacio público o para toda la zona de estudio, ya sea como un promedio o como un total. Es importante señalar que en caso de los indicadores de oferta-demanda es factible asociarlos con niveles de servicio o comodidad (TRB, 2010) o rangos recomendables respecto al distanciamiento social.

\section{Aplicación de la metodología a un estudio de caso}

Se aplica esta metodología al caso de un centro histórico de una ciudad de tamaño poblacional medio de México, entre 500000 y 999999 habitantes, con traza reticular en la que confluyen en horarios matutinos para realizar sus actividades diarias. Los centros históricos en México se caracterizan por la concentración de uso de suelo mixto en el que se amalgaman, en forma intensiva, actividades laborales, servicios públicos, una amplia estructura de comercios, abastecimiento, turismo y oficinas administrativas. En ellos puede llegar a confluir el 90\% de servicios de transporte público urbano, suburbano y foráneo.

La delimitación del centro histórico como zona de estudio considera principalmente: a) las zonas de alta afluencia de personas asociadas con la concentración de actividades esenciales, como el abastecimiento mercados y ejes de comercio al por menor y los servicios públicos de oficinas de la administración pública, bancos; b) las zonas de alta concentración de usuarios del transporte público en la que confluyen las maniobras de ascensos y descensos, c) los entornos donde se ubican o se concentran altos volúmenes de tránsito, como son: plazas públicas, parques, zonas de esparcimiento entre otras, d) la estructura vial predominante y los sentidos de circulación para permitir el ajuste de los recorridos de las rutas de transporte público así como los desplazamientos de cruce por la zona central de los vehículos motorizados.

\section{Inventario de las características geométricas del espacio público disponible}

El inventario de información existente se integra en repositorio de Sistema de Información Geográfica. A partir de esta información, los levantamientos de campo 
actualizan las capas que lo integran. Las bases de datos con las características geométricas del espacio público a nivel de tramo de calle de cada manzana se muestran en la Tabla 2, síntesis del repositorio con la descripción de la capa y la fuente de información.

\section{Tabla 2}

\section{Componentes del inventario de características geométricas}

\begin{tabular}{|c|c|c|}
\hline $\begin{array}{l}\text { Nombre del archivo } \\
\text { geográfico }\end{array}$ & Descripción & Fuente sugerida \\
\hline Manzanas & $\begin{array}{l}\text { Polígonos con atributos e identificadores de: } \\
\text { municipio, área geoestadística, manzana y } \\
\text { tipo de población. }\end{array}$ & $\begin{array}{l}\text { Instituto Nacional de } \\
\text { Estadística y Geografía } \\
\text { [INEGI, 2016] }\end{array}$ \\
\hline Puntos Singulares & $\begin{array}{l}\text { Polígonos con atributos: nombre del } \\
\text { elemento, tipo de elemento (parque, iglesia, } \\
\text { escuela, etc.). }\end{array}$ & OpenStreetMap (s. f.) \\
\hline Equipamiento & $\begin{array}{l}\text { Polígonos con atributos: nombre del } \\
\text { elemento, clasificación del equipamiento: } \\
\text { Educación, Salud, Religión, Transporte, } \\
\text { Comercio, Mercados, Cultural, Recreación }\end{array}$ & Gobiernos Municipales \\
\hline Unidades económicas & $\begin{array}{l}\text { Puntos con atributos: identificador, nombre } \\
\text { del establecimiento, clave de la actividad, } \\
\text { actividad económica, datos del } \\
\text { establecimiento, cantidad de empleados. }\end{array}$ & $\begin{array}{l}\text { Instituto Nacional de } \\
\text { Estadística y Geografía } \\
\text { [INEGI, 2020] }\end{array}$ \\
\hline Traza Urbana & $\begin{array}{l}\text { Polígonos sin atributos que incluye: lotes, } \\
\text { ancho de banquetas y límite de manzana. }\end{array}$ & $\begin{array}{l}\text { Patronatos, Autoridades de } \\
\text { Centros Históricos }\end{array}$ \\
\hline Red vial & $\begin{array}{l}\text { Polilíneas con atributos: clave vial, nombre de } \\
\text { la vía, tipo de vía, sentido de la vía, ámbito de } \\
\text { la vía (urbana y suburbana), jurisdicción. }\end{array}$ & $\begin{array}{l}\text { Instituto Nacional de } \\
\text { Estadística y Geografía } \\
\text { [INEGI, 2016] con } \\
\text { actualizaciones propias }\end{array}$ \\
\hline Trazo de Rutas & $\begin{array}{l}\text { Polilíneas con atributos: nombre de la ruta y } \\
\text { sentido de la ruta. }\end{array}$ & $\begin{array}{l}\text { Gobiernos de los Estados } \\
\text { con actualizaciones propias. }\end{array}$ \\
\hline $\begin{array}{l}\text { Paradas transporte } \\
\text { público }\end{array}$ & $\begin{array}{l}\text { Puntos con atributos: identificador de la } \\
\text { parada, claves de las rutas que comparten el } \\
\text { paradero. }\end{array}$ & $\begin{array}{l}\text { Gobiernos de los Estados } \\
\text { con actualizaciones propias. }\end{array}$ \\
\hline
\end{tabular}


Tabla 2. Continuación

\begin{tabular}{lll}
\hline $\begin{array}{c}\text { Nombre del archivo } \\
\text { geográfico }\end{array}$ & \multicolumn{1}{c}{ Descripción } & \multicolumn{1}{c}{ Fuente sugerida } \\
\hline $\begin{array}{l}\text { Terminales y áreas de } \\
\text { encierros }\end{array}$ & $\begin{array}{l}\text { Polígonos con atributos: identificador, } \\
\text { clasificación de tipo de encierro. }\end{array}$ & $\begin{array}{l}\text { Gobiernos de los Estados } \\
\text { con actualizaciones propias. }\end{array}$ \\
Estacionamientos & $\begin{array}{l}\text { Polilínea con atributo del tipo: público o } \\
\text { privado. }\end{array}$ & $\begin{array}{l}\text { Patronatos, Autoridades del } \\
\text { Centro Histórico }\end{array}$ \\
$\begin{array}{l}\text { Rampas para } \\
\text { discapacitados }\end{array}$ & $\begin{array}{l}\text { Punto con atributos: identificador, cuadrante } \\
\text { de ubicación, estado físico de la rampa. }\end{array}$ & $\begin{array}{l}\text { Patronatos, Autoridades del } \\
\text { Centro Histórico }\end{array}$ \\
Accesos a vehículos & Polilínea con atributos: tipo de acceso. & $\begin{array}{l}\text { Patronato del Centro } \\
\text { Histórico }\end{array}$ \\
\hline
\end{tabular}

Nota: Las fuentes citadas son genéricas e indicativas. Para cada ciudad o zona de estudio pueden ser distintas. Fuente: Elaborado por los autores. Ver INEGI $(2016,2020)$, Open Street Maps (s. f.) y Google (2020).

Para elaborar la Tipología de secciones transversales existentes se considera el ancho del arroyo vehicular y el ancho de las banquetas. En cuanto al arroyo vehicular, se define un ancho de carril mínimo recomendable para vehículos particulares y autobuses de 2.5 y 3.5 metros respectivamente. Al considerar los anchos y carriles de todos los tramos de la malla vial, se obtienen tres tipos de categorías: 2 autos, 1 auto +1 autobús, 2 autobuses. Respecto a las banquetas, se identifican una diversidad de anchos, los cuales se clasifican en menores de 90 y mayores de 90 centímetros siendo este valor la referencia como ancho suficiente para desplazarse. Estos valores se organizaron para conformar la tipología general cuya codificación se indica en la Tabla 3.

La representación espacial de las tipologías obtenidas permite identificar, por un lado, que el espacio público en la zona de estudio es bastante heterogéneo en cuanto a su geometría. En efecto, la Figura 1 muestra, a partir de las tonalidades de color, la diversidad de tipologías a lo largo de las vialidades. Se podrá notar la falta de continuidad en cuanto a la homogeneidad del espacio para la circulación. Por otro lado, existe un cierto número de secciones que carecen del espacio mínimo para la 
Tabla 3

Tipología de secciones transversales existentes

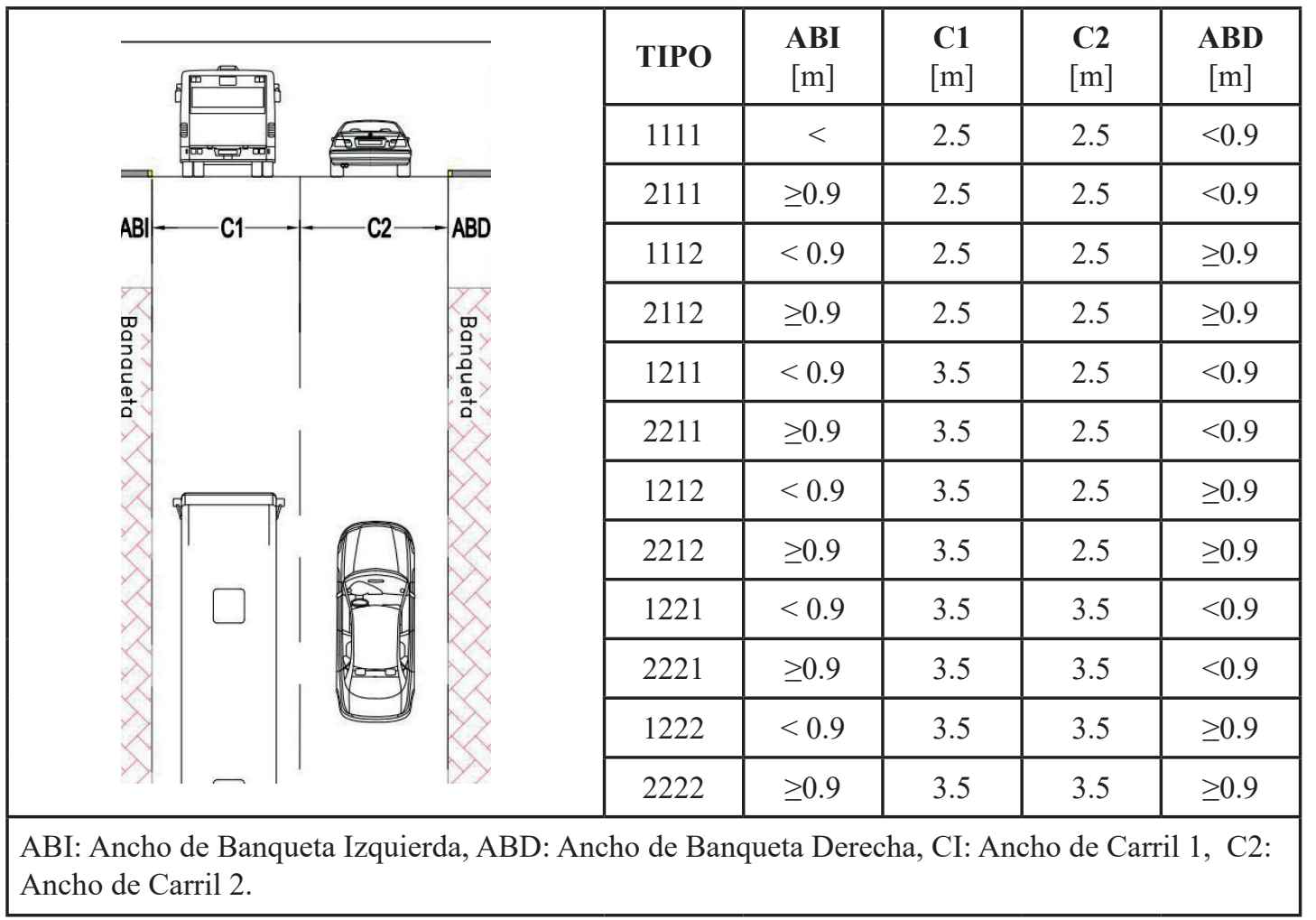

Fuente: Elaborada por los autores.

circulación. Esto implica que las condiciones para el distanciamiento social tanto para la circulación como para la formación de filas son desfavorables. Este diagnóstico es muy similar en la mayoría de los centros históricos ya que la geometría que prevalece actualmente ha sido el resultado de los antecedentes y procesos históricos como se ha planteado previamente. (Figura 1)

Se definieron las secciones tipo para el uso modal mixto del espacio público siguiendo los lineamientos planteados y considerando las características geométricas existentes para ampliar el espacio público y favorecer el distanciamiento social. Se pretende que las secciones no generen cuellos de botella para la circulación de cualquiera de los modos de transporte empleados. Para lograr este objetivo se estableció, en primer lugar, la homogeneización del área de circulación a lo largo de toda una vialidad y ampliar las banquetas en un solo margen de esta. Se verificó que es poco 


\section{Figura 1}

\section{Distribución de tipologías predominantes en la zona de intervención}

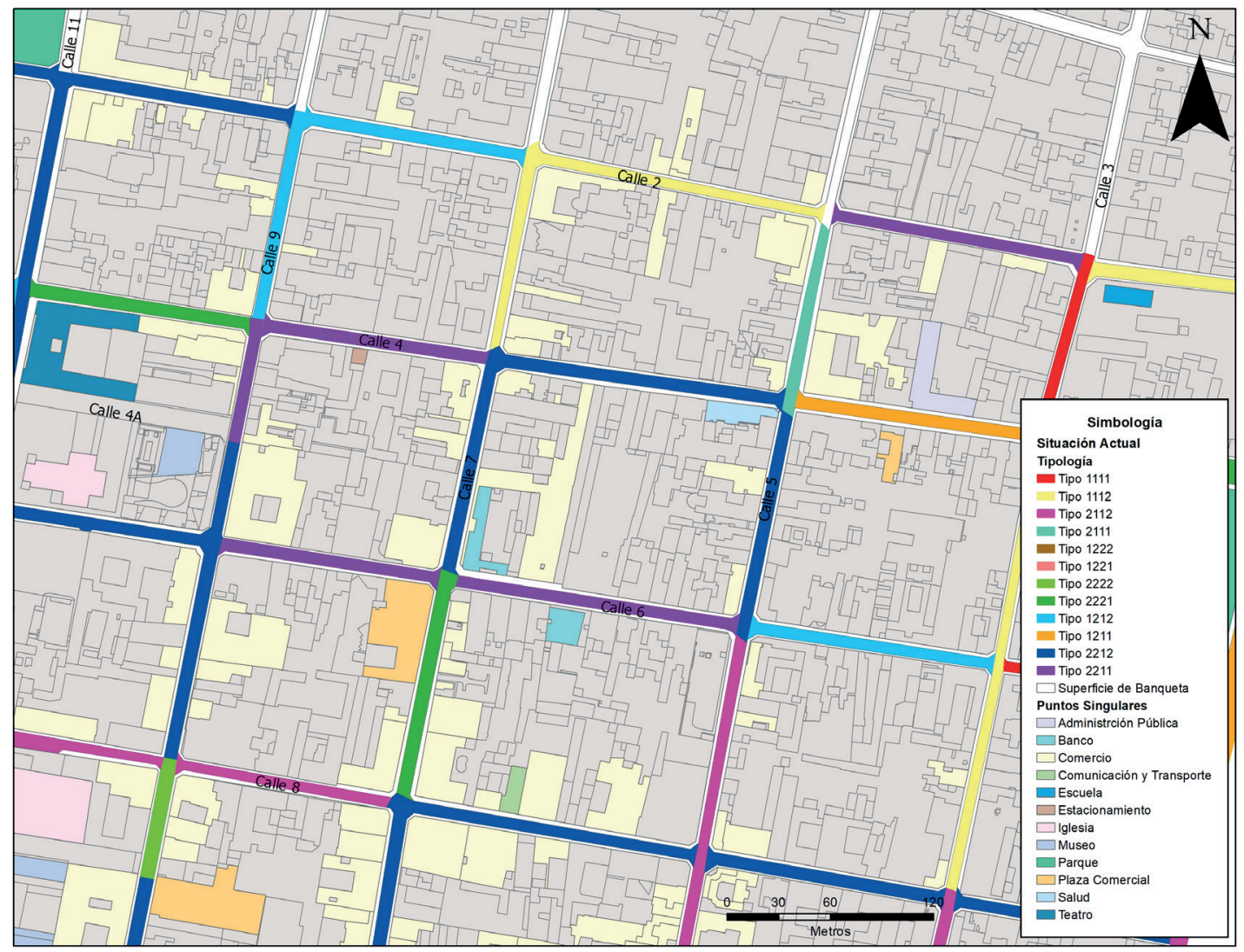

Fuente: Elaborada por los autores.

operativo ampliar en los dos costados de la vialidad cuando el ensanchamiento es menor de 60 centímetros, siendo más conveniente ampliar en un solo costado para disponer de una banqueta al menos para la circulación. En segundo lugar, para evitar eventuales conflictos de tránsito entre los peatones y los conductores de vehículos motorizados, se incorpora en el diseño de las secciones tipo un elemento físico de separación entre el arroyo vehicular y la banqueta, disuasivo para que el automovilista no invada la banqueta ampliada y al mismo tiempo ofrezca seguridad al peatón para sentirse seguro en el momento de circular en este espacio ampliado.

A partir de estas consideraciones se obtienen cuatro secciones tipo que se indican en la Tabla 4 en el orden de la jerarquía de movilidad y la prioridad modal. Las secciones propuestas se caracterizan por promover el uso modal mixto y favorecer el distanciamiento social al ampliar las banquetas. Este espacio ampliado proviene de 


\section{Tabla 4}

\section{Secciones tipo para favorecer el distanciamiento social}

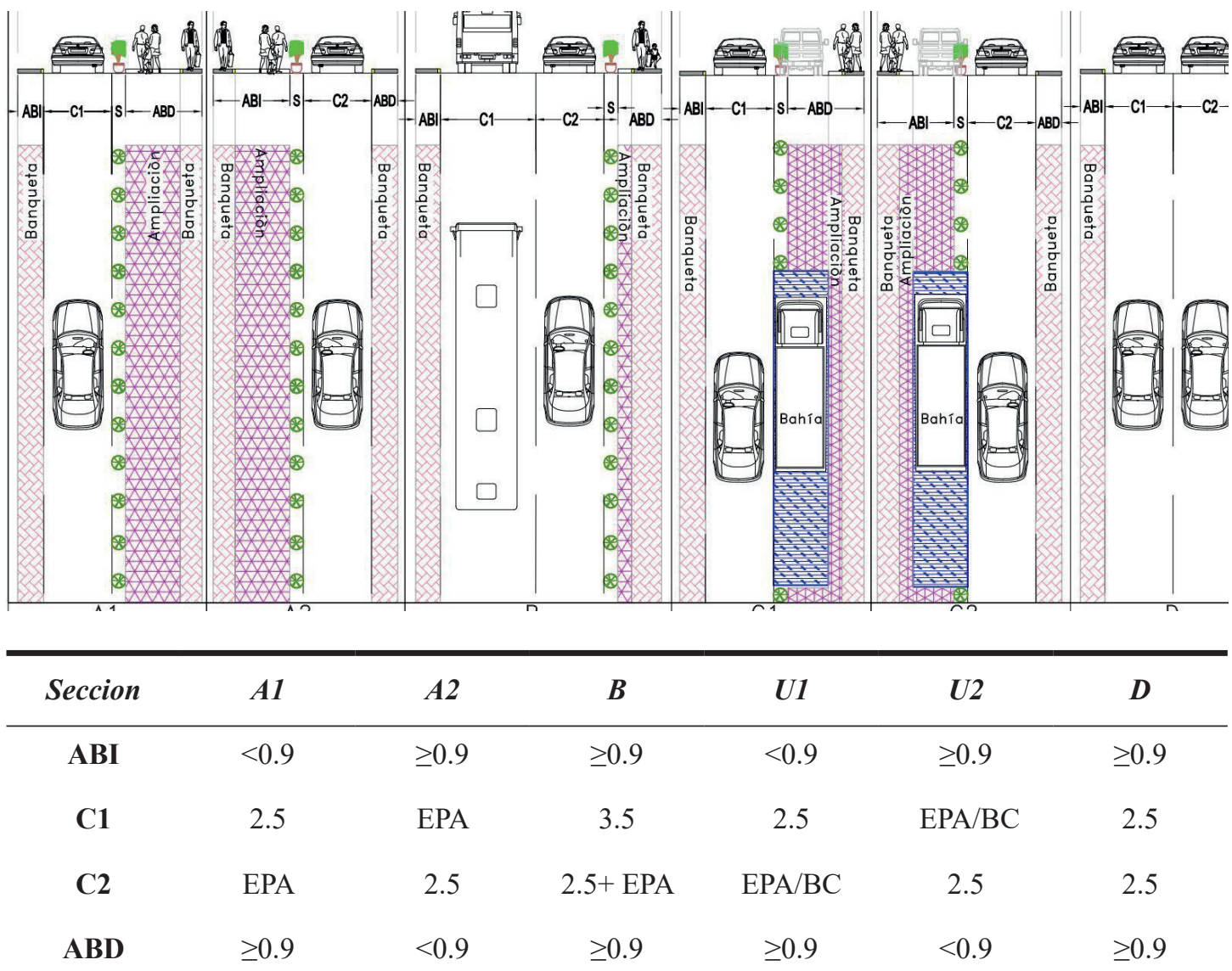

ABI: Ancho de Banqueta Izquierda, ABD: Ancho de Banqueta Derecha, C1: ancho Carril 1, C2: ancho de Carril 2, EPA: Espacio Peatonal Ampliado, BC: Bahía para Carga.

Fuente: Elaborada por los autores.

restringir el estacionamiento en la vía pública y reducir las operaciones de ascensos y descenso del transporte público. En el caso de la zona intervenida estos paraderos se reubicaron en el entorno del perímetro de intervención y se ajustó el trazo del recorrido de las rutas de transporte. Con esta medida, se redujo la carga vehicular generada por los autobuses. Así, la sección A1 y A2 según la ampliación de la banqueta sea a la derecha o la izquierda del sentido de circulación, se denomina semipeatonal (véase Tabla 4). Es la que organiza la complementariedad modal entre peatones, transporte colectivo y privado. La sección tipo B, transporte público, complementa este modo con peatones y la circulación del transporte privado. La sección 
U1 y U2, para transporte de carga, integra espacios dedicados para peatones, vehículos de carga y el transporte privado. Finalmente, la sección D, ejes viales, se complementa con el modo peatonal teniendo la función de desahogar el tránsito vehicular. En la parte inferior de la Tabla 4 se indican las dimensiones para cada tipo de sección. En los casos del Espacio Peatonal Ampliado (EPA) podrá notarse que la diversidad tipológica de la situación existente se reduce de manera notable. Con ello se atiende el objetivo de disponer de secciones homogéneas para la circulación de los diferentes modos de transporte, facilitando el flujo laminar y propiciando las condiciones para el distanciamiento social en la mayor parte del territorio intervenido.

La configuración del espacio de uso modal mixto surge a partir de las características geométricas del espacio público, se selecciona la sección tipo que sea más pertinente incorporar respecto a dos criterios. El primero establece que la sección elegida sea fácilmente implementada en una longitud lo más extensa posible de tal manera que se brinde a los usuarios, de los distintos modos de transporte, continuidad y homogeneidad en el recorrido. El segundo considera que la sección elegida sea compatible con la actividad predominante o vocación de un entorno, eje o conjunto de tramos de la malla vial. En la Figura 2 se ilustra parte de la configuración del espacio público que se obtiene como resultado, así como los espacios públicos ampliados. Se podrá notar que: a) respecto a la situación sin intervención (ver Tabla 3) las secciones tipo asignadas son más homogéneas por largas extensiones, b) es visible una correspondencia entre estas secciones tipo y las vocaciones predominantes en los ejes viales y c) en general, que el espacio público tiene una mayor organización.

Los indicadores propuestos de organización del espacio y del distanciamiento social se presentan en la Tabla 5, así como su variación porcentual entre la situación con la implementación de la metodología propuesta y sin intervención. Todos los indicadores muestran mejoría en la organización del espacio público y en las condiciones que favorecen el distanciamiento social. En más de la mitad de las vialidades se amplía el espacio para la circulación peatonal (PEPP) lo que implica un incremento de cerca de dos tercios (SCP). En cuanto a la disponibilidad de espacios para formar filas, los espacios vitales $^{1}(\mathrm{CEV})$ se incrementan en más del doble (145\%), véase Tabla 5.

${ }^{1}$ Se asume una superficie de 2 metros de distanciamiento social recomendado por 0.6 metros del ancho mínimo para una persona. 


\section{Figura 2}

\section{Configuración resultante para el uso modal mixto del espacio público}

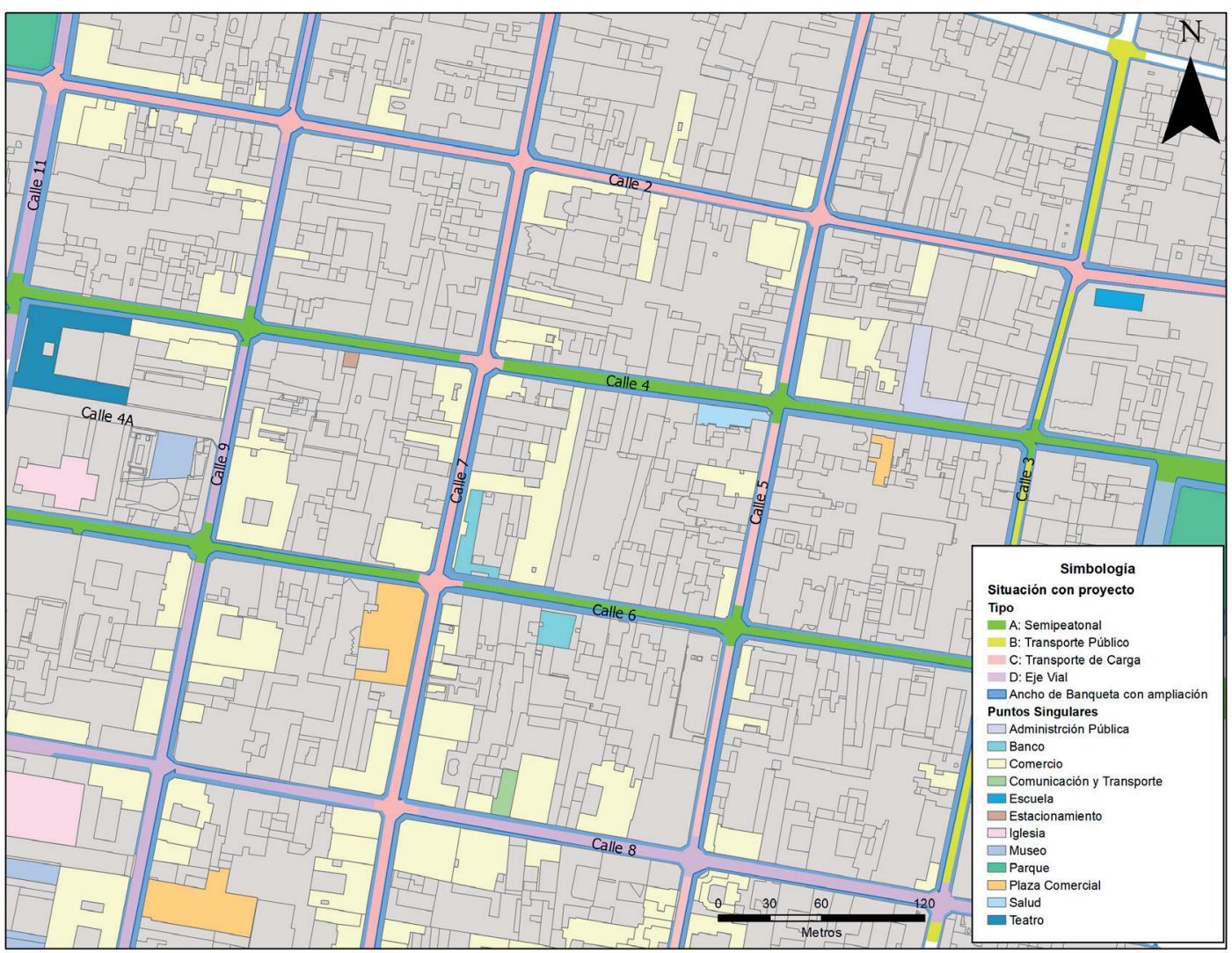

Fuente: Elaborada por los autores.

La estimación de los indicadores que relacionan oferta y demanda requiere contabilizar el número de personas que ocupan el espacio público en un periodo de tiempo determinado. Existen varias técnicas de conteo para estimarlos, como aforos peatonales en el periodo pico de la mañana y de la tarde ya que estos representan las condiciones más críticas del día teniendo en el resto de las horas del día una menor movilidad lo que llevaría a tener condiciones más favorables de distanciamiento social. Estas mediciones se realizaron el miércoles 2 de diciembre de 2020. Según la densidad y la concentración, puede notarse que la movilidad de usuarios es más importante en el período pico de la mañana. Ambos indicadores mejoran con la gestión del espacio público propuesta ya que su valor absoluto se reduce y con ello hay una mayor dispersión de las personas. La mejora en promedio para toda la zona 


\section{Tabla 5}

Indicadores de distanciamientos social situación sin y con intervención

\begin{tabular}{|c|c|c|c|c|}
\hline Indicador & Unidad & $\begin{array}{c}\text { Sin } \\
\text { intervención }\end{array}$ & $\begin{array}{l}\text { Con gestión del } \\
\text { espacio público }\end{array}$ & $\begin{array}{l}\text { Variación } \\
(X c-X s) / X s\end{array}$ \\
\hline $\begin{array}{l}\text { PEMM: Proporción de Ejes } \\
\text { Modales Mixtos }\end{array}$ & $\%$ & 0 & 64.3 & NA \\
\hline $\begin{array}{l}\text { PEPP: Proporción de espacios } \\
\text { públicos ampliados para uso } \\
\text { peatonal }\end{array}$ & $\%$ & 0 & 53.9 & NA \\
\hline $\begin{array}{l}\text { SCP: Superficie dedicada a la } \\
\text { circulación peatonal }\end{array}$ & $\mathrm{m}^{2}$ & 55450 & 92096 & $66 \%$ \\
\hline $\begin{array}{l}\text { CEV: Cantidad de Espacios } \\
\text { Vitales (CEV) para formación } \\
\text { de filas de espera con } \\
\text { distanciamiento social }\end{array}$ & $\begin{array}{l}\text { Espacios } \\
\text { vitales }\end{array}$ & 14348 & 35097 & $145 \%$ \\
\hline $\begin{array}{l}\text { DSCP: Densidad de personas en } \\
\text { las Superficies dedicadas a la } \\
\text { Circulación Peatonal (promedio) }\end{array}$ & Pers $/ \mathrm{m}^{2}$ & 0.97 & 0.72 & -25.8 \\
\hline Pico AM & Pers $/ \mathrm{m}^{2}$ & 1.05 & 0.82 & $-22 \%$ \\
\hline Pico PM & Pers $/ \mathrm{m}^{2}$ & 0.93 & 0.67 & $-28 \%$ \\
\hline $\begin{array}{l}\text { CSCP: concentración de personas } \\
\text { en las superficies dedicadas a la } \\
\text { Circulación Peatonal }\end{array}$ & Pers $* \mathrm{~m}^{2}$ & 0.25 & 0.15 & $-40 \%$ \\
\hline Pico AM & Pers $* \mathrm{~m}^{2}$ & 0.27 & 0.21 & $-22 \%$ \\
\hline Pico PM & Pers* $\mathrm{m}^{2}$ & 0.24 & 0.12 & $-50 \%$ \\
\hline $\begin{array}{l}\text { DSP: Distanciamiento } \\
\text { Social Promedio }\end{array}$ & metros & 8.7 & 21.0 & $210 \%$ \\
\hline Pico AM & metros & 7.7 & 36.6 & $374 \%$ \\
\hline Pico PM & metros & 9.3 & 19.5 & $109 \%$ \\
\hline
\end{tabular}

Fuente: Elaborado por los autores. 
intervenida es del orden del $25 \%$ para la densidad y más de $40 \%$ para la concentración. Es en el período más crítico del día que la mejora es más sustancial, siendo del orden del $50 \%$. El distanciamiento social mejora notablemente pues pasa en promedio de casi 9 a 21 metros, una mejora del 267\%. Sin embargo, los valores indicados están agregados para la zona intervenida por lo que requieren incorporar la componente espacial para identificar de forma más precisa los lugares en específico que requieren alguna intervención cuando el distanciamiento social se encuentre en valores críticos.

La Figura 3 muestra lo anterior para el indicador de distanciamiento social contrastando la situación con y sin intervención. Además de visualizar claramente la mejora de las condiciones que propicia el distanciamiento social, la imagen muestra las zonas donde existe una mayor propensión a no atender esta acción comunitaria por lo que la autoridad pudiera definir una estrategia de intervención.\}

Figura 3

Distribución espacial del distanciamiento social

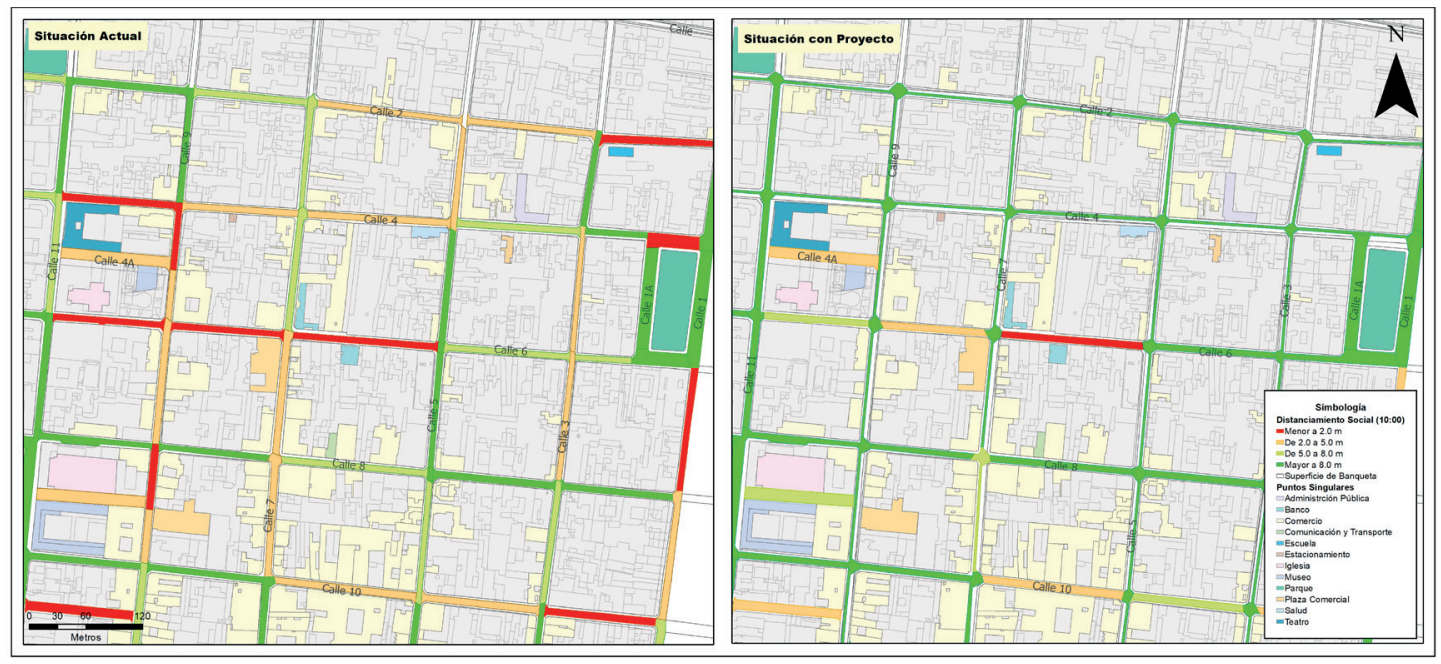

Fuente: Elaborada por los autores.

\section{Discusión y conclusiones}

La duración de la pandemia ocasionada por el coronavirus SARS-CoV2 es todavía incierta. Si bien el desarrollo de la vacuna aporta una manera de resolver 
el problema aún se requiere la aplicación a una gran parte de la población y posteriormente de un conjunto de procesos para garantizar su erradicación. Desafortunadamente transcurrirán varios meses antes que esto ocurra. Entretanto, una serie de transformaciones en los hábitos de consumo, movilidad y producción tendrán lugar. Estos cambios llevan también a replantear la reorganización del espacio público como un medio para propiciar la acción comunitaria del distanciamiento social lo que a su vez reduce el riesgo de propagación de contagio del citado virus. En este artículo, se plantea que esta reorganización se realice con un enfoque de uso modal mixto siguiendo una integración vertical basada en la jerarquía de la movilidad sostenible y horizontal en la complementariedad de los modos de transporte. Con ello, el espacio público disponible se amplía lo que propiciaría el distanciamiento social. La metodología propuesta requiere de información, elementos, herramientas y capacidades técnicas disponibles en la mayor parte de instituciones de planeación. Por lo tanto, requiere recursos relativamente bajos para implementarla. Por otro lado, la tipología y el dimensionamiento de las secciones tipo se han generalizado para facilitar su adaptación a las normas o criterios prevalecientes en cada zona de estudio. Se incluye además un conjunto de indicadores específicos para evaluar la conveniencia de las alternativas que puedan configurarse en función de las características de una zona urbana en particular. Sin duda que se podrían adicionar indicadores complementarios. Sin embargo, la restricción es la colecta de los insumos ya que la movilidad, y en general el transporte, varía en el espacio y el tiempo. En efecto, se requiere monitorear regularmente este tipo de indicadores lo que implica, por un lado, sistematizar su cálculo y por otro lado, la colecta continua de información. Los avances en la colecta de información, la disponibilidad de big data, la neogeografía y geografía individual integran el desarrollo de herramientas sistemáticas (Bosque Sendra, 2015) de seguimiento del distanciamiento social, pero sobre todo para la intervención y la reducción del riesgo de contagio.

El análisis empírico presentado permite comprobar las ventajas comparativas que ofrece la reorganización del espacio público propuesto a través de esta metodología. La magnitud de estas dependerá de las características geométricas, de la configuración existente de los modos de transporte y las particularidades de cada zona de intervención. Un aspecto relevante para tomar en cuenta en la implementación de estas medidas es la inclusión de los actores del espacio público con la finalidad 
de aminorar los eventuales efectos negativos. Finalmente, se requiere de una campaña de comunicación efectiva respecto al proceso de cambio a fin de aportar certeza y confianza a la sociedad en su conjunto propiciando con ello el respeto y cumplimiento de estas acciones comunitarias.

\section{Referencias bibliográficas}

Balaguer, Pedro. (2018). “Geografía crítica y pensamiento críticos”. Actualidades Pedagógicas, (72). 73-95. https://doi.org/10.19052/ap.5232

BorJa, Jordi; Muxí, Zaida. (2003). El espacio público: ciudad y ciudadanía. Electa/diputación. Bosque Sendra, Joaquín. (2015). "Neogeografía, Big Data y TIG: problemas y nuevas posibilidades". Polígonos, Revista de Geografia, (27), 165-174. http://doi.org/10.18002/ pol.v0i27.3277

Coraggio, José Luis. (1987). Territorios en transición, crítica a la planificación en América Latina. Centro de Investigaciones ciudad.

FitzPatrick, Key; Wooldridge, Mark; BlaschKe, Joseph. (2005). Urban Intersection Design Guide (Volume 1 - Guidelines). Texas Transportation Institute.

Google. (2020). "Google Community Mobility reports" [Base de datos de reportes de movilidad para México]. Recuperado el 29 de diciembre 2020 de https://www.google.com/covid19/ mobility

Hiernaux, Daniel; Lindón, Alicia. (1993). "El concepto de espacio y el análisis regional" Secuencia, (25), 89-110. https://doi.org/10.18234/secuencia.v0i25.411

Instituto Nacional de Estadística y Geografía (INEGi). (2016). Inventario Nacional de Viviendas 2016. INEGI.

Instituto Nacional de Estadística y Geografía (inegi). (2020). Directorio Estadístico Nacional de Unidades Económicas. DENUE Interactivo 04/2020. INEGI.

Instituto de Políticas para el Transporte y el Desarrollo (itdP). (2012). Planes Integrales de Movilidad Lineamientos para una movilidad urbana sustentable. ITDP.

Lazcano, Eduardo; Alpuche, Celia. (2020). "Alfabetización en salud pública ante la emergencia de la pandemia por Covid-19”. Revista Salud Publica de México, 62(3), 331340. https://doi.org/10.21149/11408

Moovit Insights. (s. f.). "Estadísticas de transporte público por país y por ciudad" (en línea). Moovit. Recuperado 23 marzo 2021 de https://moovitapp.com/insights/es/Moovit_ Insights_\%C3\%8Dndice_de_Transporte_P\%C3\%BAblico-countries?utm_source $=$ seo_ maps

Moraes, Antonio. (1984). Geografia: pequena história crítica. Hucitec. 
National Association of City Transportation Officials (nacto). (2013). "Urban Street Design Guide". NACTO. Recuperado el 13 de diciembre de 2020 de https://nacto.org/ publication/urban-street-design-guide/

OpenStreetMap. (s. f.). "Proyecto de mapeo de México" (en línea). OpenStreetMap. Recuperado el 23 de marzo de 2021 de en https:/wiki.openstreetmap.org/wiki/ ES:M\%C3\%A9xico

SCHLACK, Elke. (2007). "Espacio público". ARQ (Santiago), (65), 25-27. https://dx.doi. org/10.4067/S0717-69962007000100006

Secretaría de Desarrollo Agrario, Territorial y Urbano (Sedatu). (2019). Manual de calles: Diseño vial para ciudades mexicanas. SEDATU. Recuperado 16 de marzo 2021 de https://www.gob.mx/sedatu/documentos/manual-de-calles-diseno-vial-para-ciudadesmexicanas .

Stamatiadis, Nikiforos; Kirkm, Adam. (2010). Improving Intersection Design Practices. Kentucky Transportation Center.

Transportation Research Board (TrB). (2010). Highway Manual Capacity. Trb. 